\title{
EVALUATION OF CROP-DAIRY PRODUCTION SYSTEM IN NILE DELTA
}

\author{
S.M. Alsheikh ${ }^{1}$, Salah Galal ${ }^{2}$, M.N. Rashwan ${ }^{3}$ and Samira A. Arafa ${ }^{3}$ \\ 1- Desert Research Center, Mataria, Cairo, Egypt, 2- Faculty of Agriculture, Ain \\ Shams University, Shubra Alkhamia, Cairo, Egypt, 3- Animal Production Research \\ Institute, Dokki, Giza, Egypt
}

\section{SUMMARY}

A linear programming (LP) model was applied to evaluate crop-dairy production system in small mixed farms in the Nile Delta through the impact of Food Sector Development Program (FSDP) of the Ministry of Agriculture and Land Reform. The LP model considered land, labor, large ruminant genotypes, cropping pattern and available cash resources (ACR) as factors affecting production of this system. Technical coefficients of the LP model were estimated from a survey data collected from 492 farmers in five different governorates, Damietta (DAM), Daqahleia (DAQ), Gharbeia (GHA), Kafr El-Sheikh (KEL) and Menoufeia (MEN), during the period from 1993 to 2001. Two districts were sampled within each governorate, one with farmers collaborating with FSDP, who got at least one training package $\left(C_{1}\right)$ and another with non-collaborating farmers (receiving no training packages (control group)) $\left(C_{0}\right)$. The objective function of LP model was to determine the optimum combination of crops and dairy production, which maximizes the gross margin (GM) of the farm, which enhances the return per feddan (RPF) and the return per animal (RPA). One LP model with three runs was tested. The first run (base run) was to simulate the actual situation, the second $\left(L P_{1}\right)$ to avoid the unacceptable solution for the base run while the third $\left(\mathrm{LP}_{2}\right)$ to get feasible solution for the district that had no feasible solution. Results suggested that, in base run solution, farmers in general should combine cultivating berseem in winter with rice in summer plus keeping buffalo before FSDP package adoption. While, after FSDP package adoption the farmers should combine cultivating cash crops with berseem in winter, rice in summer and crossbred cattle in district $C_{1}$ in MEN and in both district in DAQ, while other districts should keep exotic cattle. The FSDP improved GM, RPF and RPA by about $9 \%, 5 \%$ and $11 \%$, respectively in base run, by about $10 \%, 7 \%$ and $10 \%$, respectively, in $L P_{1}$ and by about $8 \%, 3 \%$ and $10 \%$, respectively in $L P_{2}$. In conclusion LP model showed that, both land and available cash resources were the limiting resources while labor was not. In mixed dairy production system in Nile delta, dairy animal activities contribute substantially, about $25 \%$, to the total farm gross margin.

Keywords: Linear programming, small mixed farm, cattle, buffalo, Egypt

Issued by The Egyptian Society of Animal Production 


\section{INTRODUCTION}

In Egypt, the crop-dairy production system is quite common in many regions, i.e. Nile delta and valley and newly reclaimed lands. This system harbors livestock producing $90 \%$ of the total milk production (Abdel-Aziz, 1997). Eight five percent of the total domestic milk output is provided by traditional cropping-dairy farms and $15 \%$ by the dairy-specialized commercial sector (MoALR, 2004).

Food Sector Development Program (FSDP), which is considered in the present study, lasted from 1991 to 2001 and was funded by the European Commission (EC) for a value of 9.9 million Euro and executed by the Ministry of Agriculture and Land Reform. FSDP activities were servicing the dairy sector and focused on the production, processing and marketing of milk. Also, FSDP included technical components, supporting the development of the dairy sector. FSDP activities were institution building, training and demonstration, technical assistance and a 75 million Euro fund as credit and guarantee scheme. Target group was the small and medium scale farmers.

The objective of this study was to evaluate cropping-dairy production system in small mixed farms in the Nile delta through the impact of FSDP.

\section{MATERIAL AND METHODS}

Data:

A survey was conducted using an objective questionnaire. Data were collected on 492 crop-dairy farms between 1993 and 2001 as a part of the FSDP to develop the cropping-dairy farming system in the Nile delta. The data were collected only from farmers who practice cropping plus buffaloes and/or one or more dairy cattle genotypes, i.e. native (Baladi) and exotic cattle and crosses between them. Each farm had two interviews, one at the beginning of the program during 1993 to 1997 and another at the end during 1998 to 2001. Five governorates in the Nile delta were involved, Damietta (DAM), Daqahleia (DAQ), Gharbeia (GHA), Kafr El-Sheikh $(\mathrm{KEl})$ and Menoufeia (MEN). Two districts were sampled from each governorate, one had collaborating $\left(\mathrm{C}_{1}\right)$ farmers and another that had no collaborating $\left(\mathrm{C}_{0}\right)$ farmers. Collaborating farmers would get at least one of the FSDP training packages while non-collaborating farmers received no such training packages (as a control group). Training packages included 15 training programs on how farmers can increase their dairy animal productivity, produce clean milk and improve farm income. Table 1 shows the distribution of farmers.

Table 2 shows available resources, cropping pattern, and dairy animal production before and after intervention through the FSDP adopted training package(s) in dairy animal activities excluding small ruminants due to their small number.

\section{Mathematical LP model}

The LP model considered land, labor, large ruminant genotypes, cropping pattern and available cash resources (ACR) as factors affecting crop/dairy production system. This model is applied to determine the optimum combination of crops with dairy, which maximizes the gross margin (GM) of the farm and leads to increase the return per animal (RPA) and the return per feddan $(\mathrm{RPF})\left(1 \mathrm{feddan}=4200 \mathrm{~m}^{2}\right)$. One LP model with three runs was performed, the first run (base run) to simulate the 
actual situation, the second $\left(\mathrm{LP}_{1}\right)$ to avoid the unacceptable solution for the base run (cultivated only berseem, Trifolium alexandrinum, in winter and green fodder in summer). While the third $\left(\mathrm{LP}_{2}\right)$ was to get feasible solution for the district(s) that had no feasible solution. Input estimates of the model were analyzed using General Algebra Modeling System (GAMS, 2000) software. The model structure for the three runs were as follows:

Table 1. Number of farmers in two districts within each of the five studied governorates

\begin{tabular}{|c|c|c|c|}
\hline Governorate & District & Number of farmers & Total \\
\hline \multirow[t]{3}{*}{ Damietta } & & & 73 \\
\hline & $\mathrm{C}_{0}$-Kafr Saad & 26 & \\
\hline & $\mathrm{C}_{1}-$ Faraskur & 47 & \\
\hline \multirow[t]{3}{*}{ Daqahleia } & & & 103 \\
\hline & $\mathrm{C}_{0}$-Sherbeen & 12 & \\
\hline & $\mathrm{C}_{1}$-Senbllewien & 91 & \\
\hline \multirow[t]{3}{*}{ Gharbeia } & & & 104 \\
\hline & $\mathrm{C}_{0}$-Tanta & 20 & \\
\hline & $\mathrm{C}_{1}$-Quttur & 84 & \\
\hline \multirow[t]{3}{*}{ Kafr-El-Sheikh } & & & 87 \\
\hline & $\mathrm{C}_{0}$-Dessouk & 8 & \\
\hline & $\mathrm{C}_{1}-\mathrm{Q}$ allin & 79 & \\
\hline \multirow[t]{3}{*}{ Menoufeia } & & & 125 \\
\hline & $\mathrm{C}_{0}$-Ashmoun & 31 & \\
\hline & $\mathrm{C}_{1}$-Shanshour & 94 & \\
\hline \multirow[t]{3}{*}{ Total } & & & 492 \\
\hline & $\mathrm{C}_{0}$ & 97 & \\
\hline & $\mathrm{C}_{1}$ & 395 & \\
\hline
\end{tabular}

\section{Base run:}

Objective function: Maximize (gross margin) $=\sum_{j=1}^{12} a_{\mathrm{j}} \mathrm{X}_{\mathrm{i}}$, where,

$a_{j}$ gross margin for each variable of $X_{i}, X_{i}$ are number of feddans cultivated with berseem $\left(X_{1}\right)$, wheat $\left(X_{2}\right)$, winter cash crops $\left(X_{3}\right)$, faba bean $\left(X_{4}\right)$, rice $\left(X_{5}\right)$, summer cash crops $\left(\mathrm{X}_{6}\right)$, maize $\left(\mathrm{X}_{7}\right)$, cotton $\left(\mathrm{X}_{8}\right)$, number of buffaloes $\left(\mathrm{X}_{9}\right)$, number of native cattle $\left(\mathrm{X}_{10}\right)$, number of crossbred cattle $\left(\mathrm{X}_{11}\right)$ and number of exotic cattle $\left(\mathrm{X}_{12}\right)$.

Constraints:

Land,

Winter $\mathrm{X}_{1}+\mathrm{X}_{2}+\mathrm{X}_{3}+\mathrm{X}_{4} \leq$ average farm size

Summer $\mathrm{X}_{5}+\mathrm{X}_{6}+\mathrm{X}_{7}+\mathrm{X}_{8} \leq$ average farm size 
$\sum_{i=j=1}^{\text {Labor, }} \mathrm{c}_{\mathrm{j}} \mathrm{X}_{\mathrm{i}} \leq \mathrm{b}$,

where,

$\mathrm{c}_{\mathrm{j}}$ is labor (adult-day) requirement

$\mathrm{b}$ is total labor; and $\mathrm{X}_{\mathrm{i}}$ as before.

$$
\sum_{i=j=1}^{\mathrm{ACR},} \mathrm{d}_{\mathrm{j}} \mathrm{X}_{\mathrm{i}} \leq \mathrm{m},
$$

where,

$d_{j}$ is variable cost for each variable; $m$ available cash resources; and $X_{i}$ as before.

$$
\text { Non negativity }
$$

$$
\mathrm{X}_{\mathrm{i}} \geq 0, \quad \mathrm{i}=1, \ldots, 12 \text {. }
$$

\section{Second run $\left(L P_{1}\right)$ :}

Objective function: the same as base run.

Constraints:

$$
\text { Land, }
$$

Winter $\quad \mathrm{X}_{1}+\mathrm{X}_{2}+\mathrm{X}_{3}+\mathrm{X}_{4} \leq$ average farm size

$\mathrm{X}_{1} \geq 1$ feddan

$\mathrm{X}_{2} \geq 1$ feddan

$\mathrm{X}_{3} \geq 1$ feddan

$\mathrm{X}_{4} \geq 1$ feddan

Summer $\mathrm{X}_{5}+\mathrm{X}_{6}+\mathrm{X}_{7}+\mathrm{X}_{8} \leq$ average farm size

$\mathrm{X}_{5} \geq 1$ feddan

$\mathrm{X}_{6} \geq 1$ feddan

$$
\mathrm{X}_{7} \geq 1 \text { feddan }
$$

$\mathrm{X}_{8} \geq 1$ feddan

Labor,

$$
\sum_{i=j=1}^{12} \mathrm{c}_{\mathrm{j}} \mathrm{X}_{\mathrm{i}} \leq \mathrm{b},
$$

where,

$\mathrm{c}_{\mathrm{j}}$ is labor (adult-day) requirement

$\mathrm{b}$ is total labor; and $\mathrm{X}_{\mathrm{i}}$ as before.

ACR,

$$
\sum_{i=j=1}^{12} \mathrm{~d}_{\mathrm{j}} \mathrm{X}_{\mathrm{i}} \leq \mathrm{m},
$$

where,

$d_{j}$ is variable cost for each variable; $m$ available cash resources; and $X_{i}$ as before. Non negativity

$$
\mathrm{X}_{\mathrm{i}} \geq 0, \quad \mathrm{i}=1, \ldots, 12 .
$$


Table 2. Raw means of available resources before and after adopting FSDP training packages

\begin{tabular}{|c|c|c|c|c|c|c|c|c|c|c|}
\hline & \multicolumn{2}{|c|}{ DAM } & \multicolumn{2}{|c|}{ KEL } & \multicolumn{2}{|c|}{ MEN } & \multicolumn{2}{|c|}{ DAQ } & \multicolumn{2}{|c|}{ GHA } \\
\hline & $\mathrm{C}_{1}$ & $\overline{\mathrm{C}_{0}}$ & $\mathrm{C}_{1}$ & $\mathrm{C}_{0}$ & $\overline{\mathrm{C}_{1}}$ & $\mathrm{C}_{0}$ & $\overline{\mathrm{C}_{1}}$ & $\mathrm{C}_{0}$ & $\overline{\mathrm{C}_{1}}$ & $\mathrm{C}_{0}$ \\
\hline \multicolumn{11}{|c|}{ Average farm size (feddan) } \\
\hline & 5.4 & 3.1 & 8.9 & 4.8 & 6.4 & 2.3 & 10.1 & 5.5 & 8.8 & 5.2 \\
\hline & $(5.7)$ & $(3.1)$ & $(9.9)$ & $(4.8)$ & $(6.8)$ & $(2.3)$ & $(10.6)$ & $(5.5)$ & $(9.0)$ & $(5.1)$ \\
\hline \multicolumn{11}{|c|}{ Labor (person day)/farm per year } \\
\hline & 616 & 528 & 615 & 352 & 528 & 616 & 738 & 528 & 704 & 861 \\
\hline \multirow[t]{2}{*}{ Winter } & $(528)$ & $(528)$ & $(492)$ & $(352)$ & $(528)$ & $(738)$ & $(861)$ & $(616)$ & $(792)$ & (1107) \\
\hline & 581 & 516 & 590 & 472 & 498 & 357 & 708 & 516 & 944 & 602 \\
\hline Summer & $(516)$ & $(516)$ & $(472)$ & $(472)$ & $(708)$ & $(516)$ & $(826)$ & $(602)$ & $(1062)$ & $(774)$ \\
\hline \multicolumn{11}{|c|}{ Winter cropping area (feddan) } \\
\hline & 3.0 & 1.1 & 3.5 & 1.9 & 2.5 & 1.0 & 4.1 & 2.6 & 3.6 & 3.2 \\
\hline \multirow[t]{2}{*}{ Berseem } & $(3.0)$ & $(1.1)$ & $(3.6)$ & $(2.9)$ & $(2.9)$ & $(1.0)$ & $(5.6)$ & $(2.6)$ & $(3.7)$ & $(3.1)$ \\
\hline & 1.4 & 1.0 & 3.3 & 1.8 & 2.0 & 0.5 & 2.5 & 1.5 & 2.0 & 1.5 \\
\hline Wheat & $(1.7)$ & $(1.0)$ & $(2.3)$ & $(1.9)$ & $(2.0)$ & $(0.5)$ & $(3.0)$ & $(1.5)$ & $(3.0)$ & $(1.5)$ \\
\hline Cash & 1.0 & 1.0 & 2.1 & - & 1.9 & 0.8 & 2.5 & 1.4 & 2.0 & 1.5 \\
\hline crops & $(1.0)$ & $(1.0)$ & $(2.0)$ & $(-)$ & (1.9) & $(0.8)$ & $(2.0)$ & $(1.4)$ & $(2.3)$ & $(1.5)$ \\
\hline Faba & - & - & - & 1.1 & - & - & 1.0 & - & 1.2 & - \\
\hline bean & $(-)$ & $(-)$ & $(2.0)$ & $(-)$ & $(-)$ & $(-)$ & $(-)$ & $(-)$ & $(-)$ & $(-)$ \\
\hline \multicolumn{11}{|c|}{ Summer cropping area (feddan) } \\
\hline \multirow[t]{2}{*}{ Rice } & 3.0 & 1.1 & 3.1 & 1.9 & - & - & 4.1 & 2.6 & 3.6 & 3.2 \\
\hline & $(2.1)$ & (1.1) & (3.6) & (1.9) & $(-)$ & $(-)$ & (4.6) & $(2.6)$ & (3.7) & (3.1) \\
\hline Cash & 1.0 & 1.0 & 1.2 & - & 2.0 & 1.3 & 1.0 & 1.5 & 1.2 & 1.5 \\
\hline crops & $(0.6)$ & (1.0) & $(2.0)$ & $(-)$ & $(2.0)$ & (1.3) & $(1.0)$ & (1.5) & (1.3) & (1.5) \\
\hline \multirow[t]{2}{*}{ Maize } & 1.4 & 1.0 & 1.6 & 1.8 & 2.5 & 1.0 & 2.5 & 1.4 & 2.0 & 1.5 \\
\hline & $(2.0)$ & (1.0) & $(2.0)$ & (1.8) & (2.9) & (1.0) & $(2.0)$ & (1.4) & (1.0) & (1.5) \\
\hline \multirow[t]{2}{*}{ Cotton } & - & - & 3.0 & 1.1 & 1.9 & - & 2.5 & - & 2.0 & - \\
\hline & $(1.0)$ & $(-)$ & (2.1) & (1.1) & (1.9) & $(-)$ & $(3.0)$ & $(-)$ & $(3.0)$ & $(-)$ \\
\hline \multicolumn{11}{|c|}{ Average herd size (head) } \\
\hline & 1.3 & 1.2 & 1.5 & 2.0 & 2.4 & 1.5 & 1.4 & 2.1 & 1.7 & 1.2 \\
\hline Buffalo & $(1.3)$ & $(1.0)$ & $(1.2)$ & $(2.0)$ & $(2.5)$ & $(1.5)$ & $(1.4)$ & $(2.0)$ & $(4.7)$ & $(1.2)$ \\
\hline \multirow[t]{3}{*}{ Baladi } & 0.6 & 0.5 & 0.8 & 0.8 & 0.5 & 0.4 & 0.5 & 0.7 & 0.5 & 0.7 \\
\hline & $(0.2)$ & $(0.2)$ & $(0.4)$ & $(0.5)$ & $(-)$ & $(-)$ & $(0.2)$ & $(0.4)$ & $(-)$ & $(-)$ \\
\hline & 0.4 & 0.5 & 1.0 & 1.3 & 1.2 & - & 0.7 & 1.1 & 1.0 & 1.3 \\
\hline \multirow[t]{2}{*}{ Crossbred } & $(0.6)$ & $(0.6)$ & $(1.1)$ & $(2.0)$ & $(0.5)$ & $(0.6)$ & $(0.7)$ & $(1.3)$ & $(-)$ & $(2.0)$ \\
\hline & 0.2 & - & 0.6 & - & - & 0.2 & 0.8 & 1.0 & 1.5 & 1.0 \\
\hline Exotic & $(0.6)$ & $(0.5)$ & $(0.7)$ & $(-)$ & $(0.6)$ & $(-)$ & $(0.8)$ & (1.3) & $(-)$ & $(1.0)$ \\
\hline
\end{tabular}

Third run $\left(L P_{2}\right)$ :

Objective function: the same as base run Constraints:

Land,

Winter $\quad \mathrm{X}_{1}+\mathrm{X}_{2}+\mathrm{X}_{3}+\mathrm{X}_{4} \leq$ average farm size

$\mathrm{X}_{1} \geq 0.75$ feddan

$\mathrm{X}_{2} \geq 0.75$ feddan

$\mathrm{X}_{3} \geq 0.75$ feddan

$\mathrm{X}_{4} \geq 0.75$ feddan 
Summer $\mathrm{X}_{5}+\mathrm{X}_{6}+\mathrm{X}_{7}+\mathrm{X}_{8} \leq$ average farm size

$\mathrm{X}_{5} \geq 0.75$ feddan

$X_{6} \geq 0.75$ feddan

$\mathrm{X}_{7} \geq 0.75$ feddan

$\mathrm{X}_{8} \geq 0.75$ feddan

Labor,

$$
\sum_{i=j=1}^{12} \mathrm{c}_{\mathrm{j}} \mathrm{X}_{\mathrm{i}} \leq \mathrm{b} \text {, }
$$

where,

$c_{j}$ is labor (adult-day) requirement

$\mathrm{b}$ is total labor; and $\mathrm{X}_{\mathrm{i}}$ as before.

$\mathrm{ACR}$,

$$
\sum_{i=j=1}^{12} \mathrm{~d}_{\mathrm{j}} \mathrm{X}_{\mathrm{i}} \leq \mathrm{m},
$$

where,

$\mathrm{d}_{\mathrm{j}}$ is variable cost for each variable; $m$ available cash resources; and $\mathrm{X}_{\mathrm{i}}$ as before. Non negativity

$$
\mathrm{X}_{\mathrm{i}} \geq 0, \quad \mathrm{i}=1, \ldots, 12 .
$$

\section{RESULTS AND DISCUSSION}

\section{LP (base run) solution:}

LP (base run) solution shown in Table 3 suggests that, farmers in all districts should cultivate berseem in winter and rice in summer, except Sherbeen in DAQ $\left(\mathrm{C}_{0}\right)$ where the solution suggested that farmers should cultivate cash crops beside berseem in winter and rice in summer. While in both districts in MEN (Shanshour and Ashmoun) farmers should cultivate berseem in winter and cash crops in summer. Moreover, the solutions suggested that farmers before FSDP should keep buffalo in all governorates except both district in DAM governorate where the solutions suggested that farmers should keep crossbred cattle. While after FSDP, the solution suggested that farmers should keep crossbred cattle in district $\mathrm{C}_{1}$ in MEN and in both district in DAQ, while other districts should keep exotic breeds. This result indicates that the FSDP impacted dairy animal activities. Also, DAQ is traditionally a rice growing governorate, when a farmer goes to dairy production, he does so by raising crossbreed, exotic or buffalo and grows more of fodder crops

The solutions of the base run (Table 3) found the highest GM and RPA in $C_{1}$ in $\mathrm{KEl}$ in both states of FSDP intervention. Also, the solutions showed that GM, RPA and RPF, in general, showed positive FSDP impact, where differences between after and before FSDP within all $\mathrm{C}_{1}$ districts were higher than the differences between after and before FSDP within all $\mathrm{C}_{0}$ districts. This result could possibly occur due to FSDP credit and guarantee and intervention packages schemes available to $C_{1}$ farmers. Results of base run also showed that dairy animal activities contributed considerably to the total GM, representing about $25 \%$, which is greater than those reported by Ahmed (1995) of about $20 \%$ for dairy animals in newly reclaimed lands. GM, RPF and RPA increased in general by about $9 \%, 5 \%$ and $11 \%$, respectively after FSDP. 
Table 3. LP (base run) solution for the two districts within five governorates before and after FSDP impact

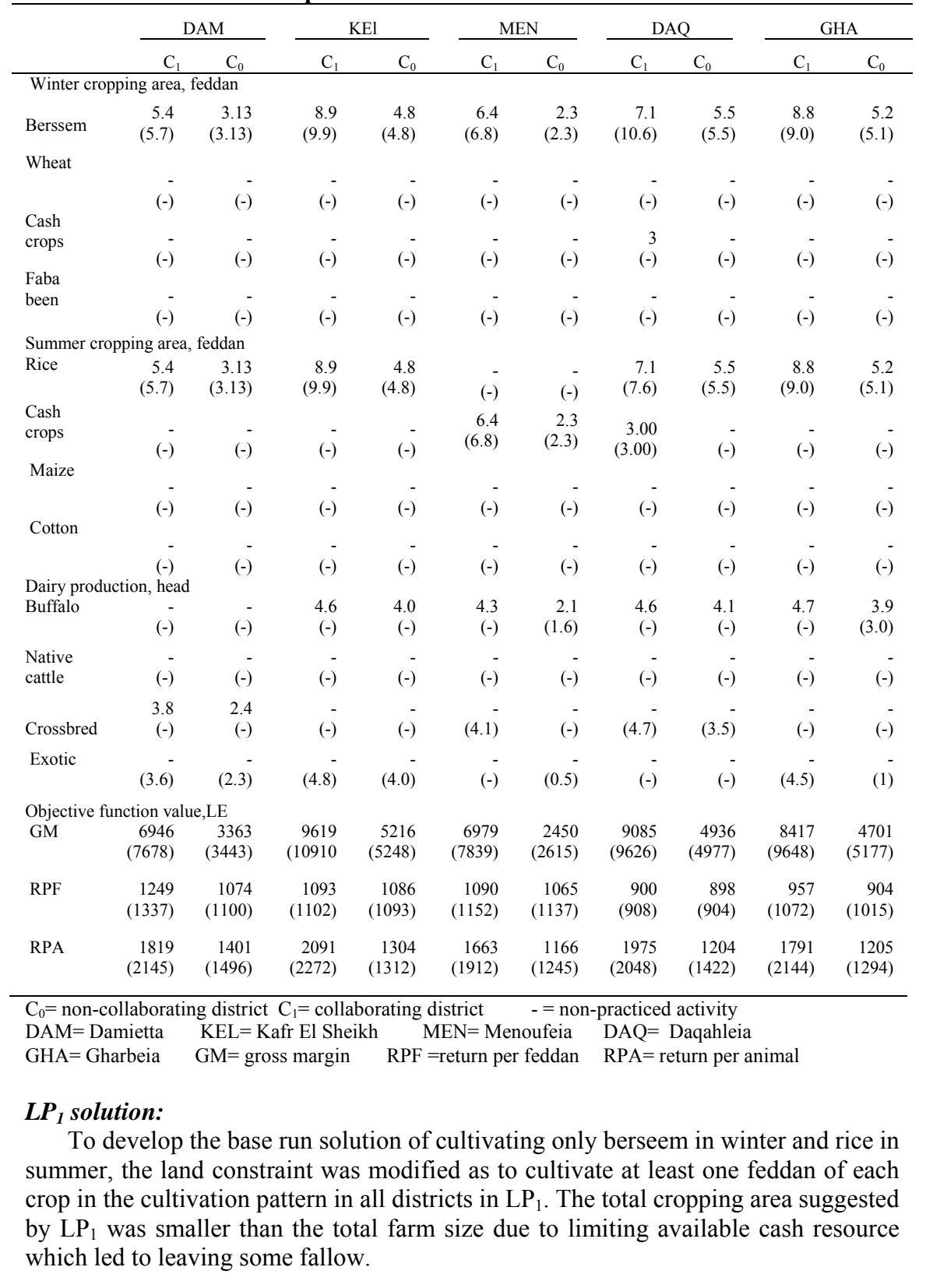


Moreover, the solution (Table 4) suggested that, farmers before FSDP should keep buffalo in all districts, except both districts in DAM where the solution suggested they should keep crossbreeds and $\mathrm{C}_{1}$ district in GHA where farmers should keep buffalo plus exotic genotypes. While after FSDP, the solution suggested that, farmers should keep combinations of buffalo, crossbreed and exotic cattle in all districts except in both districts in DAM where farmers should keep just exotics. That might be due to the fact that DAM is known for milk processing (dairy products) that needs higher producing dairy animals for the industry (ISDC, 2002).

Table 4. $L P_{1}$ solution for the two districts in five governorates before and after FSDP impact

\begin{tabular}{|c|c|c|c|c|c|c|c|c|c|c|}
\hline & \multicolumn{2}{|c|}{ DAM } & \multicolumn{2}{|c|}{$\mathrm{K}$ El } & \multicolumn{2}{|c|}{ MEN } & \multicolumn{2}{|c|}{ DAQ } & \multicolumn{2}{|c|}{ GHA } \\
\hline & $\mathrm{C}_{1}$ & $\mathrm{C}_{0}$ & $\mathrm{C}_{1}$ & $\mathrm{C}_{0}$ & $\mathrm{C}_{1}$ & $\mathrm{C}_{0}$ & $\mathrm{C}_{1}$ & $\mathrm{C}_{0}$ & $\mathrm{C}_{1}$ & $\mathrm{C}_{0}$ \\
\hline \multicolumn{11}{|c|}{ Winter cropping area, feddan } \\
\hline Berssem & $\begin{array}{r}3.3 \\
(3.5)\end{array}$ & $\begin{array}{r}1 \\
(1)\end{array}$ & $\begin{array}{r}4.2 \\
(6.0)\end{array}$ & $\begin{array}{r}1.8 \\
(1.8)\end{array}$ & $\begin{array}{r}3.8 \\
(4.3)\end{array}$ & & $\begin{array}{r}4.4 \\
(4.4)\end{array}$ & $\begin{array}{r}3.5 \\
(2.9)\end{array}$ & $\begin{array}{r}5 \\
(5.3)\end{array}$ & $\begin{array}{r}3 \\
(2.7)\end{array}$ \\
\hline Wheat & $\begin{array}{r}1 \\
(1)\end{array}$ & $\begin{array}{r}1 \\
(1)\end{array}$ & $\begin{array}{r}1 \\
(1)\end{array}$ & $\begin{array}{r}1 \\
(1)\end{array}$ & $\begin{array}{r}1.2 \\
(1.1)\end{array}$ & $\stackrel{0}{\stackrel{0}{\Xi}}$ & $\begin{array}{r}1.5 \\
(1.5)\end{array}$ & $\begin{array}{r}1 \\
(1)\end{array}$ & $\begin{array}{r}1 \\
(1)\end{array}$ & $\begin{array}{r}1 \\
(1.3)\end{array}$ \\
\hline $\begin{array}{l}\text { Cash } \\
\text { crops }\end{array}$ & $\begin{array}{r}1 \\
(1)\end{array}$ & $\begin{array}{r}1 \\
(1.3)\end{array}$ & $\begin{array}{l}2.8 \\
(1)\end{array}$ & $\begin{array}{r}1 \\
(1)\end{array}$ & $\begin{array}{r}1 \\
(1)\end{array}$ & $\frac{\omega}{0}$ & $\begin{array}{r}2.3 \\
(2.3)\end{array}$ & $\begin{array}{r}1 \\
(1)\end{array}$ & $\begin{array}{r}1 \\
(1)\end{array}$ & $\begin{array}{r}1 \\
(1)\end{array}$ \\
\hline $\begin{array}{l}\text { Faba } \\
\text { been }\end{array}$ & $(-$ & $(-)$ & $(-$ & $\begin{array}{r}1 \\
(1)\end{array}$ & $(-)$ & 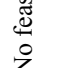 & $\begin{array}{r}1 \\
(1)\end{array}$ & $(-)$ & $\begin{array}{r}1 \\
(1)\end{array}$ & $(-$ \\
\hline \multicolumn{11}{|c|}{$\begin{array}{l}\text { Summer cropping area, } \\
\text { feddan }\end{array}$} \\
\hline Rice & $\begin{array}{r}1 \\
(1)\end{array}$ & $\begin{array}{r}1 \\
(1)\end{array}$ & $\begin{array}{r}3 \\
(4)\end{array}$ & $\begin{array}{r}1 \\
(1)\end{array}$ & $(-)$ & & $\begin{array}{r}3.0 \\
(3.0)\end{array}$ & $\begin{array}{l}2.4 \\
(1)\end{array}$ & $\begin{array}{r}4 \\
(4.3)\end{array}$ & $\begin{array}{r}1.7 \\
(1.7)\end{array}$ \\
\hline $\begin{array}{l}\text { Cash } \\
\text { crops }\end{array}$ & $\begin{array}{r}3.3 \\
(3.5)\end{array}$ & $\begin{array}{r}1 \\
(1.3)\end{array}$ & $\begin{array}{r}2.2 \\
(2.0)\end{array}$ & $\begin{array}{l}1.8 \\
(1)\end{array}$ & $\begin{array}{r}2.8 \\
(2.9)\end{array}$ & & $\begin{array}{r}2 \\
(2)\end{array}$ & $\begin{array}{l}1.1 \\
(1)\end{array}$ & $\begin{array}{r}2 \\
(2.3)\end{array}$ & $\begin{array}{r}1.3 \\
(1.3)\end{array}$ \\
\hline Maize & $\begin{array}{r}1 \\
(1)\end{array}$ & $\begin{array}{r}1 \\
(1)\end{array}$ & $\begin{array}{l}1.8 \\
(1)\end{array}$ & $\begin{array}{r}1 \\
(1.8)\end{array}$ & $\begin{array}{r}2 \\
(2)\end{array}$ & & $\begin{array}{r}2.2 \\
(2.2)\end{array}$ & $\begin{array}{r}1 \\
(1)\end{array}$ & $\begin{array}{r}1 \\
(1)\end{array}$ & $\begin{array}{r}1 \\
(1)\end{array}$ \\
\hline Cotton & $(-)$ & $(-)$ & $\begin{array}{r}1 \\
(1)\end{array}$ & $\begin{array}{r}1 \\
(1)\end{array}$ & $\begin{array}{r}1.2 \\
(1.5)\end{array}$ & & $\begin{array}{r}1 \\
(1)\end{array}$ & $(-)$ & $\begin{array}{r}1 \\
(1)\end{array}$ & (-) \\
\hline \multicolumn{11}{|c|}{ Dairy production, head } \\
\hline Buffalo & $(-)$ & $(-)$ & $\begin{array}{r}4.6 \\
(1.3)\end{array}$ & $\begin{array}{r}4.1 \\
(-)\end{array}$ & $\begin{array}{r}4.2 \\
(0.5)\end{array}$ & & $\begin{array}{r}4.6 \\
(1.2)\end{array}$ & $\begin{array}{r}4.0 \\
(1.2)\end{array}$ & $\begin{array}{r}1.5 \\
(1.2)\end{array}$ & $\begin{array}{r}3.7 \\
(3.9)\end{array}$ \\
\hline $\begin{array}{l}\text { Native } \\
\text { cattle }\end{array}$ & $(-)$ & $(-)$ & $(-)$ & $(-)$ & $(-)$ & & $(-)$ & $(-)$ & $(-)$ & $(-)$ \\
\hline Crossbred & $\begin{array}{r}3.6 \\
(-)\end{array}$ & $\begin{array}{r}2.5 \\
(-)\end{array}$ & $(-)$ & $(-)$ & (3.4) & & (3.5) & (2.6) & $(-)$ & (-) \\
\hline Exotic & (3.7) & (2.6) & (3.6) & (3.9) & $(-)$ & & (-) & (-) & $\begin{array}{r}3.1 \\
(3.3)\end{array}$ & (-) \\
\hline \multicolumn{11}{|c|}{ Objective function value, LE } \\
\hline GM & $\begin{array}{r}7.70 \\
(8003)\end{array}$ & $\begin{array}{r}3730 \\
(3962)\end{array}$ & $\begin{array}{r}9803 \\
10956\end{array}$ & $\begin{array}{r}5750 \\
(5918)\end{array}$ & $\begin{array}{r}7116 \\
(7930)\end{array}$ & & $\begin{array}{r}9706 \\
(10304)\end{array}$ & $\begin{array}{r}5082 \\
(5599)\end{array}$ & $\begin{array}{r}8640 \\
(9811)\end{array}$ & $\begin{array}{r}5035 \\
(5505)\end{array}$ \\
\hline RPF & $\begin{array}{r}1334 \\
(1455)\end{array}$ & $\begin{array}{r}1191 \\
(1265)\end{array}$ & $\begin{array}{r}1252 \\
(1370)\end{array}$ & $\begin{array}{r}1198 \\
(1233)\end{array}$ & $\begin{array}{r}1186 \\
(1239)\end{array}$ & & $\begin{array}{r}1055 \\
(1120)\end{array}$ & $\begin{array}{r}924 \\
(1018)\end{array}$ & $\begin{array}{r}1080 \\
(1182)\end{array}$ & $\begin{array}{r}1007 \\
(1101)\end{array}$ \\
\hline RPA & $\begin{array}{r}1911 \\
(2163)\end{array}$ & $\begin{array}{r}1492 \\
(1524)\end{array}$ & $\begin{array}{r}2131 \\
(2236)\end{array}$ & $\begin{array}{r}1402 \\
(1509)\end{array}$ & $\begin{array}{r}1694 \\
(2033)\end{array}$ & & $\begin{array}{r}2110 \\
(2192)\end{array}$ & $\begin{array}{r}1270 \\
(1473)\end{array}$ & $\begin{array}{r}1878 \\
(2180)\end{array}$ & $\begin{array}{r}1259 \\
(1311)\end{array}$ \\
\hline \multicolumn{11}{|c|}{$\mathrm{C}_{0}=$ non-collaborating district $\overline{C_{1}=\text { collaborating district }}-=$ no } \\
\hline $\begin{array}{l}\mathrm{DAM}=\mathrm{Da} \\
\mathrm{GHA}=\mathrm{Gh}\end{array}$ & $\begin{array}{l}\text { ietta } \\
\text { beia }\end{array}$ & $\begin{array}{l}K E L=K a \\
M=\text { gros }\end{array}$ & $\begin{array}{l}\text { El Sheik } \\
\text { margin }\end{array}$ & $\begin{array}{r}\mathrm{Ml} \\
\mathrm{RPF}=\mathrm{r}\end{array}$ & $\begin{array}{l}N=\text { Meno } \\
\text { turn per } f\end{array}$ & $\begin{array}{l}\text { offeia } \\
\text { eddan }\end{array}$ & $\begin{array}{l}\mathrm{DAQ}=\mathrm{I} \\
\mathrm{RPA}=\mathrm{re}\end{array}$ & $\begin{array}{l}\text { aqahleia } \\
\text { Irn per an }\end{array}$ & $\mathrm{mal}$ & \\
\hline
\end{tabular}




\section{$\mathrm{LP}_{2}$ solution}

To avoid the result of no feasible solution in Ashmoun $\left(\mathrm{C}_{0}\right)$ in $\mathrm{LP}_{1}$, the imposed constraint was modified to cultivate at least 0.75 feddan of each eight crops in the cultivation pattern in all districts. $\mathrm{LP}_{2}$ solution (Table 5) suggested that total cropping area was smaller than the total farm size, which led to leaving some fallow. The solution suggested that farmers should keep the same genotypes as $\mathrm{LP}_{1}$ but in different ratio.

Table 5. $\mathrm{LP}_{2}$ Solution for the two districts in five governorates before and after FSDP impact

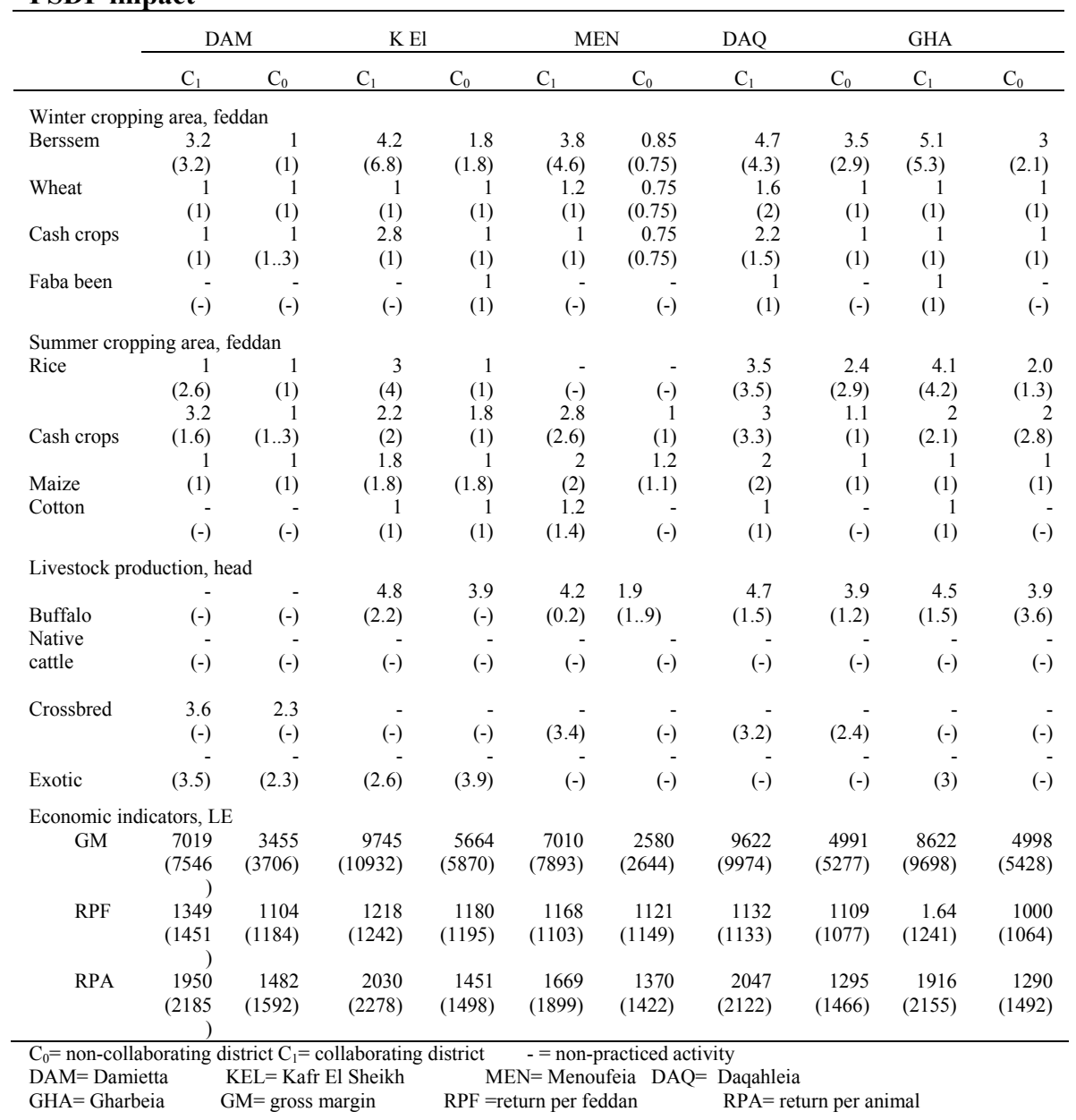

Also, $\mathrm{LP}_{2}$ solution showed that, the dairy animal activities contribution slightly increased from $25 \%$ in base run and $24 \%$ in $\mathrm{LP}_{1}$ to about $26 \%$ to the total farm gross margin. This could be due to modifying the land constraint (to cultivate at lest 0.75 
feddan) which led to decreased cultivated cropping area and increased the dairy animal activities.

GM, RPF and RPA in $\mathrm{LP}_{2}$ increased in general by about $8 \%, 3 \%$ and $10 \%$, respectively after FSDP impact and by $3 \%, 10 \%, 5 \%$ than the base run and by $2 \%$, 10 , and $9 \%$ than $L P_{1}$, respectively.

\section{CONCLUSION}

LP model with three runs showed that, both land and available cash resources were the limiting resources while labor was not. In mixed dairy production system in Nile delta, dairy animal activities contribute substantially, about $25 \%$, to the total farm gross margin. LP analysis showed that implementation of FSDP could cause a shift from farmers raising mainly buffaloes and/or native cattle only to farmers raising buffaloes plus cross breed or raising exotic breeds.

\section{REFERENCES}

Abdel-Aziz, A.S., 1997. Recent developments in the livestock sector in Egypt. A report submitted to AGA, FAO, Rome. pp 53.

Ahmed, A.M., 1995. Efficiency of Some Livestock Production Systems under Egyptian Agricultural Conditions, Ph. D. Thesis, Faculty of Agriculture, Cairo University, $255 \mathrm{pp}$.

Dillon, J.L. and J.R. Anderson, 1984. Concept and practice of farming systems research. In Proceedings of the Eastern Africa-ACIAR Consultation on Agricultural Research: Major Agricultural Problems and Research Priorities in the Eastern-Africa Region; Nairobi, Kenya, 19-22 July 1983, 171-186.

GAMS, 2000. General Algeria Modeling System software, version. 2.5, GAMS Development Corporation, 1217 Potomac St, N W Washington, DC 20007, USA.

ISDC, 2002. Describing Egypt Through Information, Information Support Decision Center, Ministerial Cabinet. Vol. 5: 334 (Arabic)

MoLAR, 2004. Agricultural Statistics, Economic Affairs Sector. Ministry of Agriculture and Land Reclamation, Egypt. pp 223.

Spedding, C.R.W., 1988. An Introduction to Agricultural Systems. Second Edition; Elsevier Applied Science. Wageningen University, Wageningen, The Netherlands. pp 400. 


\section{تقييم منظومة إنتاج المحاصيل- الحيوانات الحلابة في دلتا النيل}

سمير محمد الثيخ'، صلاح جلال، ريهام محمد نور الدين رشوان3، سميرة عبده عرفه3

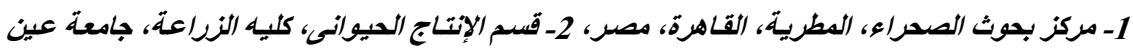

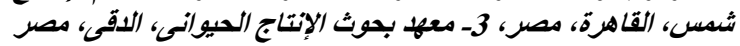

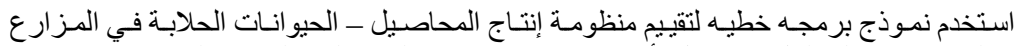

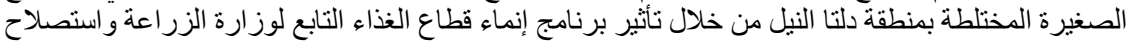

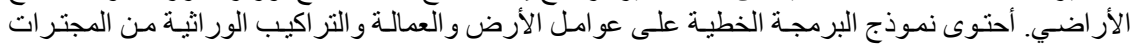

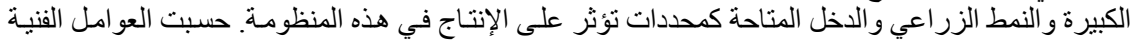

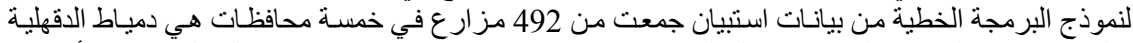

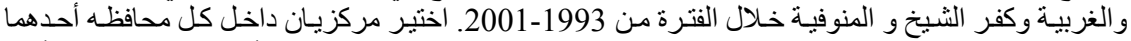

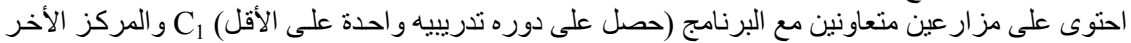

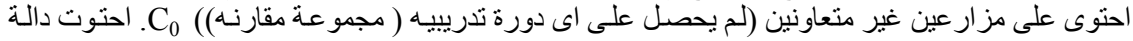

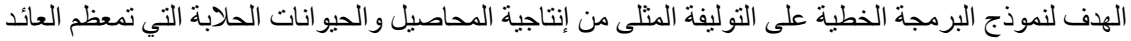

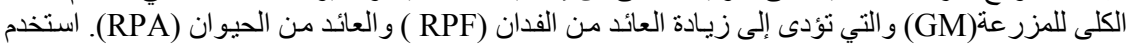

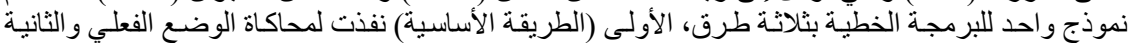

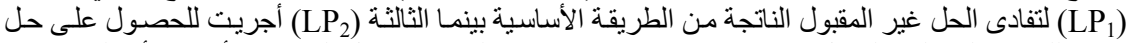

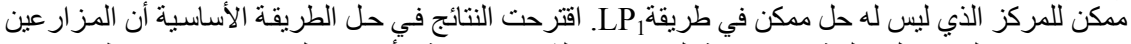

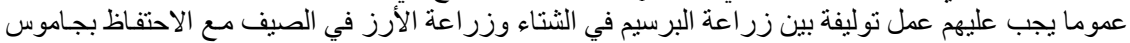

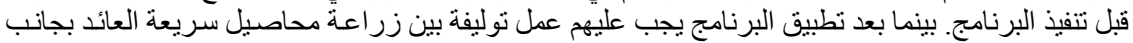

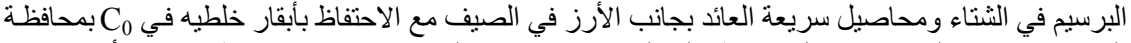

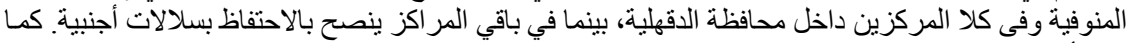

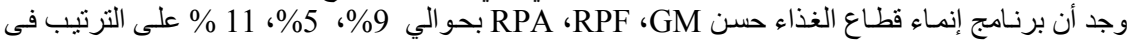

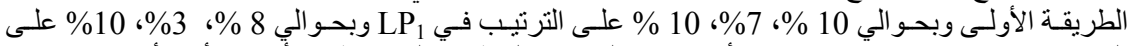

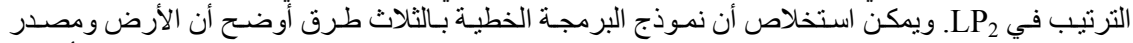

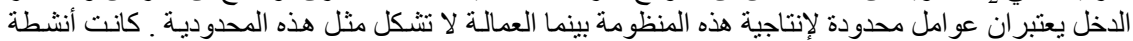

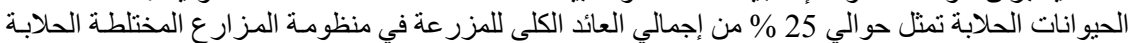
في دلتا النيل. 